\title{
A NEW CURRENT TRANSFORMER MODEL
}

\author{
Francisco das Chagas Fernandes Guerra* \\ chagasedee.ufcg.edu.br \\ Wellington Santos Mota* \\ wsmotaddee.ufcg.edu.br \\ *DEE-UFCG - Av. Aprígio Veloso, 882 - Bodocongó CEP 58109-970 - Campina Grande PB
}

\section{ABSTRACT}

This paper presents a current transformer model that is useful on low frequency applications. To describe the iron core magnetic behaviour of the iron core, a hysteresis model is proposed, which is able to generate minor asymmetric loops and remanent flux. The effects of classic eddy current losses and anomalous losses are represented by linear and nonlinear resistors, respectively. The obtained results are compared with those calculated by Preisach's model and measured in laboratory. This model may be applied in power system protection studies, as it is the case of numeric correction of distorted secondary currents in current transformers (CT's).

KEYWORDS: Current transformers, hysteresis, magnetic losses, digital protection.

\section{RESUMO}

This paper presents a current transformer model that is useful on low frequency applications. To describe the iron core magnetic behaviour of the iron core, a hysteresis model is proposed, which is able to generate minor asymmetric loops and remanent flux. The effects of classic eddy current losses and anomalous losses are represented by linear and nonlinear resistors, respectively. The obtained results are compared with those calculated by Preisach's model and measured in laboratory. This model may be applied in power system protection studies, as it is the case of numeric correction of distorted secondary currents in current transformers (CT's).

Artigo submetido em 25/04/2005

1a. Revisão em 22/06/2005;

2a. Revisão em 06/09/2005;

Aceito sob recomendação do Ed. Assoc. Prof. José Antenor Pomilio
PALAVRAS-CHAVE: Current transformers, hysteresis, magnetic losses, digital protection.

\section{INTRODUCTION}

The fault currents in electric power systems present a sinusoidal component and a DC component with exponential decline. The first produces a sinusoidal magnetic flux in the current transformer core. The second produces an initially increasing flux with subsequent decline. The total flux, associated to the remanent flux, can produce a high saturation level in the core. This fact usually causes severe distortions in the secondary currents supplied to the protection relays. In consequence, the following problems can take place:

a. The relays can operate inadequately.

b. The relays are not sensitized due to the distortions that reduce the RMS value of the secondary current.

c. The relay operations can be delayed, for the reason mentioned in the previous item.

d. The fault locators do not show correct indication.

Those occurrences can cause thermal and electrodynamics damages $(b, c)$, loss of coordination in the protection relays and difficulty of location of the faulted point (a, b, c, d), or loss of system stability $(b, c)$.

In literature, there are many works related to influences of the CT's distorted secondary currents, as well as the consequences of those distortions in the performance of the protection relays. However, in these works, the iron core nonlinear 
properties are not represented by accurate models. The magnetization characteristics are usually represented by simple models, using the saturation curve.

The magnetic hysteresis representation needs to be investigated, because it determines the values of residual flux in the CT magnetic core. Such phenomenon is more important to be taken into account in the case of non-gapped CT's, used in schemes with automatic reclosing.

The works that consider the hysteresis effects in CT's use models in which important nonlinear effects are not represented. The model proposed by Poljak and Kolibas (1988) is not able to reproduce the real magnetization properties of the ferromagnetic materials.

In other works, studies of the CT's transient behavior using an EMTP model have been made (Conrad and Oeding, 1987; Kezunovic et al, 1994; Chaudhary et al, 1994; Kang et al, 1997). This model represents the magnetic core nonlinear behavior by a hysteretic inductor (type 96), developed by Frame et al (1982), based on some modifications made in the model proposed by Talukdar and Bailey (1976). However, it was reported inaccuracies in the obtained results, when simulations and field tests were compared.

Locci and Muscas (2001) presented a correction technique of the distorted secondary currents based on a model which takes into account hysteresis and eddy current effects. However, the hysteresis model is not able to reproduce the properties of closing and elimination of the minor loops (Tellinen, 1998). However, the model applications were limited to steady state operation.

A correction technique was presented by Bittanti et al (2001), where the primary current is estimated from measurements of secondary distorted currents, using least square technique. The iron core was modeled without consider the magnetic properties of the material.

A hysteresis model requires data not always available. There are models that require a set of points of the DC loop as input data. However, it is not common to obtain this information. The data more easily available are the magnetic induction and field intensity, characteristics of the core material, usually supplied by the manufacturer. Normally, these values are converted in magnetic flux and magnetizing current, using the geometric parameters of the core and the number of turns of the windings. For power transformers, this practice produces significant errors because, in the construction of the core, the sheets are cut, having insertion of unknown gap lengths. Because of this, the obtained magnetic characteristic is not the real characteristic (Elleuch and Poloujadoff, 1998). Fortunately, such fact does not occur in case of current transformers, which present simple geometry and rela-

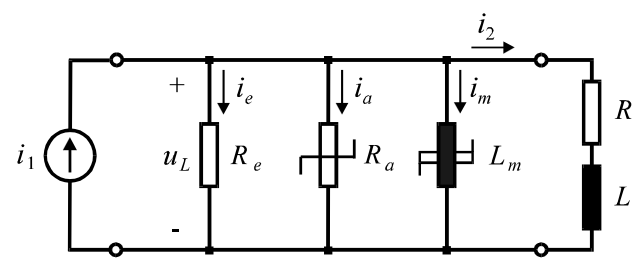

Figure 1: The proposed CT equivalent circuit.

tive easiness of data obtainment. A CT core usually has a toroidal form (a ribbon made of a silicon-iron alloy, coiled in a helicoidal way). The gaps intentionally inserted have known equivalent length.

\section{THE CURRENT TRANSFORMER MODEL}

\subsection{Equivalent Electric Circuit}

The proposed CT equivalent electric circuit is shown in figure. 1. $R$ and $L$ represent the resistance and the inductance of the secondary (winding and load). The nonlinear inductor is associated to the saturation and hysteresis effects of the magnetic core. The effects of classic eddy current losses and anomalous losses are represented by $R_{e}$ (linear resistor) $R_{a}$ (nonlinear resistor), respectively.

\subsection{Hysteresis Modeling}

The proposed model starts from the Talukdar and Bailey work (Talukdar and Bailey, 1976). A minor loop between the points $P_{k-1}$ and $P_{k-2}$ and a part of the descending branch of the major DC hysteresis loop are shown in figure 2. $x_{k}$ is the vertical distance from a point $P_{k}$, on descending trajectory of the minor loop, to the point $P_{k}$, , on descending trajectory of the major loop. It is supposed that $x_{k}$ varies as follows:

$$
x_{k}=x_{k-2}+\left(x_{k-1}-x_{k-2}\right)\left[\frac{\lambda_{k}-\lambda_{k-2}}{\lambda_{k-1}-\lambda_{k-2}}\right]^{1+\beta\left|\lambda_{k} / \lambda_{s}\right|}
$$

$x_{k-1}$ and $x_{k-2}$ are the vertical distances from $P_{k-1}$ and $P_{k-2}$ (turning points) to the descending branch of the major hysteresis loop; $\lambda_{k}, \lambda_{k-1}$ and $\lambda_{k-2}$ are the values of flux linkages in the points $P_{k}, P_{k-1}$ and $P_{k-2} ; \beta$ is a parameter that depends on the core material; $\lambda_{s}$ is the value of the flux linkages when the magnetic core starts to saturate.

The descending branch of the major loop is fitted by the function $i_{m}=G(\lambda)$. For the points $P_{k}$ and $P_{k}$, it follows that:

$$
i_{m k}=g\left(\lambda_{k}\right)=G\left(\lambda_{k}+x_{k}\right)
$$




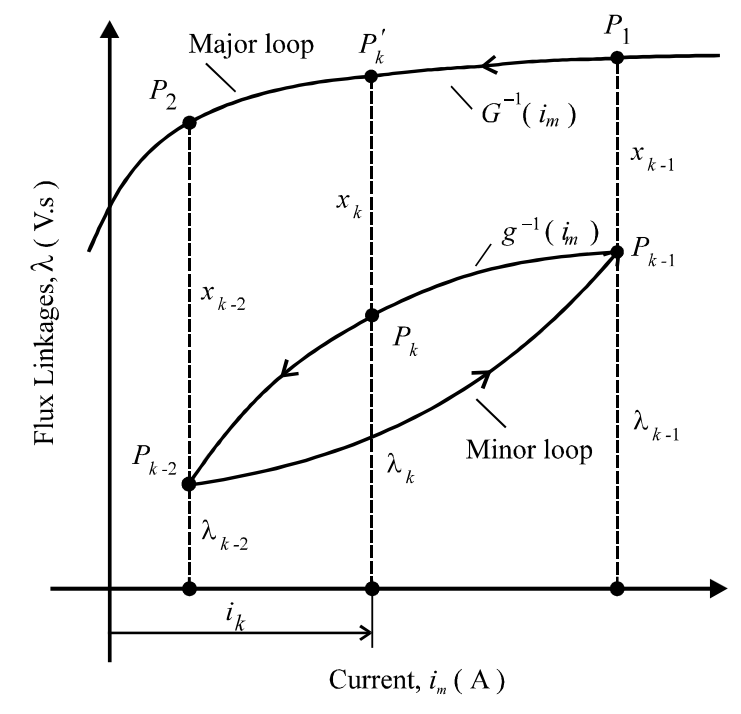

Figure 2: Asymmetric hysteresis loops.

The $\beta$ parameter establishes different slopes for the magnetic trajectories. The best value of $B$ is obtained from comparisons of magnetic paths generated by equation (1) and equation (2) with paths obtained by laboratory measurements.

Similar way is applied to the generation of ascending paths inside the major loop, considering the ascending branch.

The closing and elimination rules of the minor loops are reproduced by a stack that registers the magnetic history of the material. Initially, the stack is assumed empty. When the magnetizing trajectory passes a reversal point, this point is placed on the upper position of the stack. If the absolute value of the last calculated $\lambda$ exceeds the absolute value of the last but one value kept in the stack, a minor loop is closed, and the coordinates of the two last reversal points are removed from the stack; so, the minor loop does not have any influence in the future magnetic state of the material.

In order to represent the major hysteresis loop, the following function may be used:

$$
i_{m}=a \cdot \tan \left(\frac{\lambda}{b}\right) \pm c
$$

The constants $a, b$ and $c$ are determined from the major hysteresis loop, using curve fitting routines.

In order to approximate the initial magnetization curve in the first quadrant, the following equation is used:

$$
i_{m}=a \cdot \tan \left(\frac{\lambda}{b}\right)+c\left(1-e^{-\alpha \lambda}\right)
$$

The parameter $\alpha$ reproduces the portion of the curve closest to the origin (Rayleigh region).

When the core reaches high saturation levels, equation (3) and equation (4) may be inaccurate. A way to improve the fitting is to use sequences of points of the initial magnetization curve and the major hysteresis loop. A value of $i_{m}$ corresponding to an intermediate value of $\lambda$ is obtained using a search routine in an ordinate table (Press et al, 1986) and linear interpolation. In this case, it is not necessary to determine a value for $\alpha$.

\subsection{Core Losses Modeling}

For a flux density $B=B_{m} \sin 2 \pi f t$, the total specific magnetic losses (W/kg), per cycle, is (Fiorillo and Novikov, 1990):

$$
\frac{p}{f}=\frac{p_{h}}{f}+19.7392 K_{e} B_{m}^{2} f+8.7634 K_{a} B_{m}^{3 / 2} f^{1 / 2}
$$

$f$ is the frequency and $p_{h}$ is the specific hysteresis losses (W/kg). $K_{e}$ and $K_{a}$ are, respectively, the constants of classic eddy current losses and anomalous losses.

$K_{e}, K_{a}$ and the specific hysteresis losses for cycle, $P_{h} / f$, are determined by a fitting routine, from a set of points of the curve $P / f$ versus $f$, obtained in laboratory, using a variable frequency source, with the peak induction value sustained in the nominal value of operation.

The induction $B$ in the core can be written as follows:

$$
\frac{d B(t)}{d t}=\frac{u_{L}(t)}{N S}
$$

$N$ is the number of turns of the secondary winding and $S$ is the cross-section area of iron core.

The instantaneous eddy current losses and anomalous losses in the magnetic core in figure 1 are given, respectively, for the expressions (Zhu et al, 1993):

$$
\begin{aligned}
& P_{e}(t)=K_{e}\left(\frac{d B(t)}{d t}\right)^{2} d S l \\
& P_{a}(t)=K_{a}\left|\frac{d B(t)}{d t}\right|^{3 / 2} d S l
\end{aligned}
$$

The parameter $d$ is the density of the magnetic material and $l$ is the mean magnetic path length. Replacing equation (6) in equation (7), results:

$$
P_{e}(t)=K_{e} \frac{u_{L}^{2}(t)}{N^{2} S} d l
$$

Considering that

$$
R_{e}=\frac{u_{L}^{2}(t)}{P_{e}(t)}
$$


the resistance of classic parasitic losses is:

$$
R_{e}=\frac{N^{2} S}{K_{e} d l}
$$

Replacing equation (6) in equation (8), gives:

$$
P_{a}(t)=K_{a} \frac{\left|u_{L}(t)\right|^{3 / 2}}{N^{3 / 2} S^{1 / 2}} d l
$$

The resistance of anomalous losses is:

$$
R_{a}(t)=\frac{u_{L}^{2}(t)}{P_{a}(t)}
$$

From equation (12) and equation (13), results:

$$
\begin{aligned}
R_{a}(t) & =K \sqrt{\left|u_{L}(t)\right|} \\
K & =\frac{\sqrt{N^{3} S}}{K_{a} d l}
\end{aligned}
$$

\subsection{Circuit Equations}

For the circuit in figure 1, it follows that:

$$
\begin{gathered}
\frac{d \lambda(t)}{d t}=u_{L}(t) \\
L \frac{d i_{2}(t)}{d t}+R i_{2}(t)=u_{L}(t) \\
i_{2}(t)+i_{m}(t)+i_{a}(t)+\frac{u_{L}(t)}{R_{e}}=i_{1}(t) \\
u_{L}(t)=K \sqrt{\left|u_{L}(t)\right|} i_{a}(t) \\
i_{m}(t)=g[\lambda(t)]
\end{gathered}
$$

Equation (20) describes the proposed hysteresis model. Integrating equations (16) and (17), it is obtained:

$$
\begin{gathered}
\lambda(t)-\lambda(0)=\int_{0}^{t} u_{L}(t) d t \\
L\left[i_{2}(t)-i_{2}(0)\right]+R \int_{0}^{t} i_{2}(t) d t=\int_{0}^{t} u_{L}(t) d t
\end{gathered}
$$

Using the trapezoidal integration rule, the discretized equations for the circuit are:

$$
\begin{gathered}
\lambda(\tau)-\lambda(0)=A_{1}+\frac{h}{2}\left[u_{L}(\tau)+u_{L}(\tau-h)\right] \\
L\left[i_{2}(\tau)-i_{2}(0)\right]+R\left\{A_{2}+\frac{h}{2}\left[i_{2}(\tau)+i_{2}(\tau-h)\right]\right\} \\
=A_{2}+\frac{h}{2}\left[u_{L}(\tau)+u_{L}(\tau-h)\right] \\
i_{2}(\tau)+i_{m}(\tau)+i_{a}(\tau)+\frac{u_{L}(\tau)}{R_{e}}=i_{1}(\tau) \\
u_{L}(\tau)=K \sqrt{\left|u_{L}(\tau)\right|} i_{a}(\tau) \\
i_{m}(\tau)=g[\lambda(\tau)]
\end{gathered}
$$

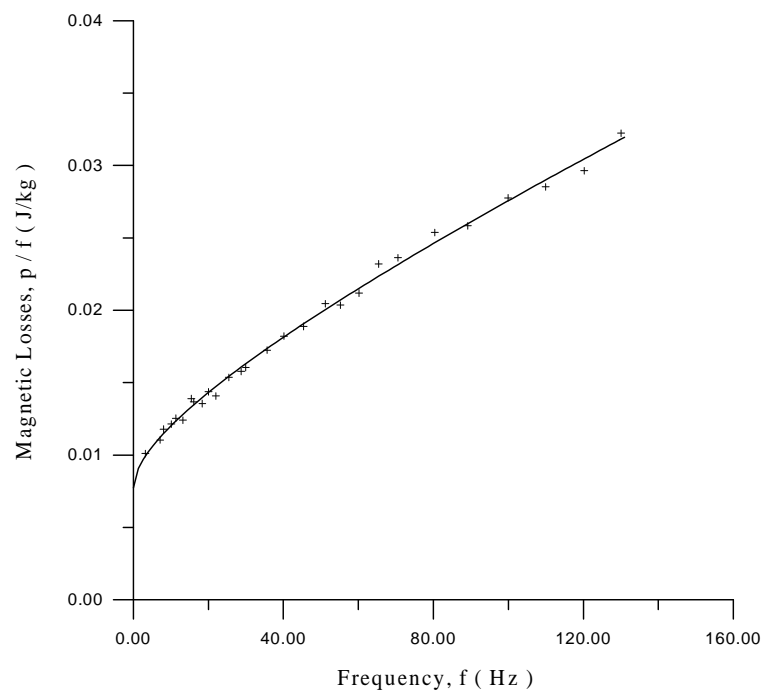

Figure 3: Magnetic losses as a function of frequency.

The constants $A_{1}$ and $A_{2}$ are given by:

$$
\begin{aligned}
& A_{1}=\int_{0}^{\tau-h} u_{L}(\tau) d \tau \\
& A_{2}=\int_{0}^{\tau-h} i_{2}(\tau) d \tau
\end{aligned}
$$

The nonlinear system is solved by using the NewtonRaphson method, considering a time step $h=1 \mu \mathrm{s}$.

\section{RESULTS}

A toroidal core was used in the laboratory measurements. It was made of a grain-oriented silicon-iron alloy, with the following data: mean diameter, $0.125 \mathrm{~m}$; cross-section area, $0.001 \mathrm{~m}^{2}$; lamination thickness, $0.3 \mathrm{~mm}$; stacking factor, 0.96 ; weight, $3 \mathrm{~kg}$; number of turns in primary and secondary windings, 60 ; windings resistance, $0.2 \Omega$.

The measured curve of magnetic losses as a function of frequency, in the range $0-130 \mathrm{~Hz}$, is presented in figure 3 , for a peak value induction $B_{m}=1.5 \mathrm{~T}$.

The parameters of equation (5) were determined using a fitting routine based on Levenberg-Marquardt's method (Press et al, 1986). The results are: $p_{h} / f=7.734 x 10^{-3} \mathrm{~J} / \mathrm{kg}, K_{e}$ $=2.076 \times 10^{-6}, K_{a}=6.590 \times 10^{-5}$.

The measured and calculated hysteresis loops are shown in figure 4 (flux linkages versus excitation current, $i=i_{m}+i_{e}$ $+i_{a}$ ), for $B_{m}=1.76 \mathrm{~T}$ and $\beta=0.3$.

The primary and secondary currents waveforms are presented in figure 5 and figure 6 , for different conditions of 


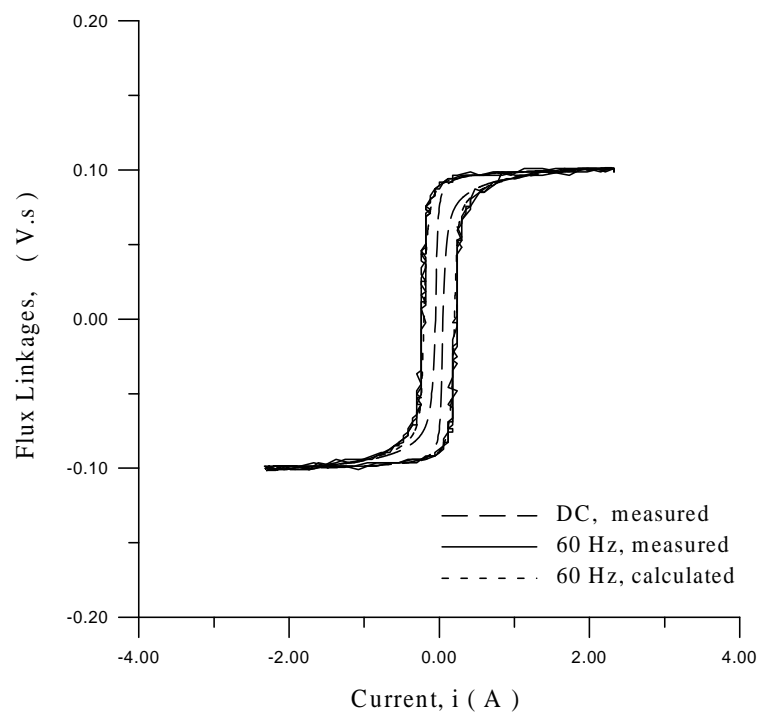

Figure 4: Hysteresis loops; $B_{m}=1.76 \mathrm{~T}, \beta=1.7$.

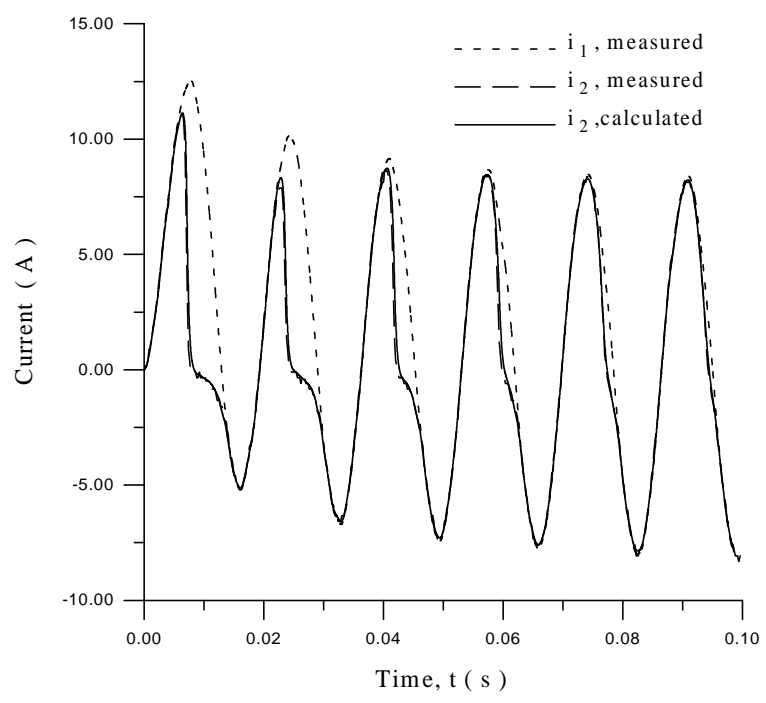

Figure 5: Currents; burden: $2.40+j 0.10 \Omega$.

excitation and burden. The excitation was increased to saturate the core, and after gradually decreased, in such way that the residual flux was made equal to zero.

The fault inception angle, $\theta$, was set for $0^{\circ}$ by a synchronous switch (a triac controlled by an electronic circuit).

The proposed CT model was compared with another model in which the hysteresis is represented by using the Preisach's theory. For this purpose, a new routine was carried out, in such way that equation (27) was replaced by $\lambda(\tau)=g^{\prime}$ [ $i_{m}(\tau)$ ], according to the formulation presented by Naidu (1990).

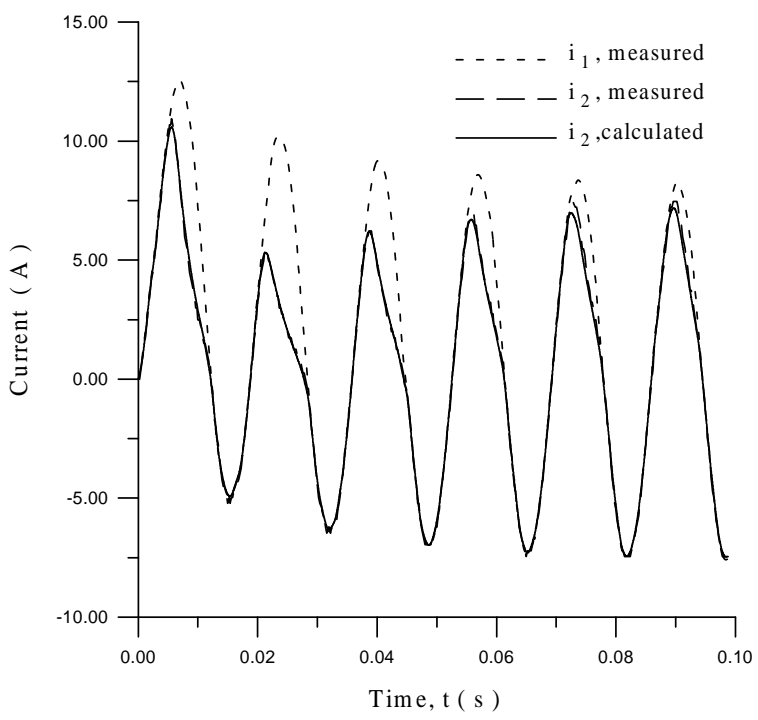

Figure 6: Currents; burden: $1.52+j 1.70 \Omega$.

Simulations were performed considering a fault at the receiving end of a hypothetic transmission line with lumped $R L$ series parameters. The transmission line impedance was 1.44 $+j 14.4 \Omega$. At the sending end, it was considered a $120 \mathrm{~V}, 60$ $\mathrm{Hz}$ voltage source. In all cases, $\lambda(0)=0$.

Hysteresis minor loops are shown in figure 7, for an impedance burden $\boldsymbol{Z}=0.25+j 0.15 \Omega$. Three intervals of time were considered: first closing, dead time and reclosing, each of them corresponding to 2 cycles in $60 \mathrm{~Hz}$, with fault inception angle $\theta=0^{\circ}$.

The flux linkages and the windings currents waveforms are shown in figure 8 and figure 9 , for a burden $\boldsymbol{Z}=1.0+j 0.5 \Omega$. It was considered closing ( 3.5 cycles), dead time $(5$ cycles $)$ and reclosing ( 5 cycles), with fault inception angles $\theta_{1}=$ (first closing) and $\theta_{2}=0^{\circ}$ (reclosing).

In order to evaluate the influence of the residual flux in the core, the fault inception angle in the reclosing period, $\theta_{2}$, was changed to $180^{\circ}$. The results are shown in figure 10 and figure 11 .

At the beginning of the reclosing period, the flux imposed by the excitation increases with same polarity than the residual flux in the core. Therefore, a higher level of saturation is reached and the distortion in the secondary current is more pronounced than those indicated in figure 9 (in this case, the flux imposed by the excitation increases with opposed polarity than the residual flux). It was observed a good agreement of the results obtained by application of the two models.

The deviation between a secondary current value given by the proposed model, $i_{2}$, and a correspondent current value 


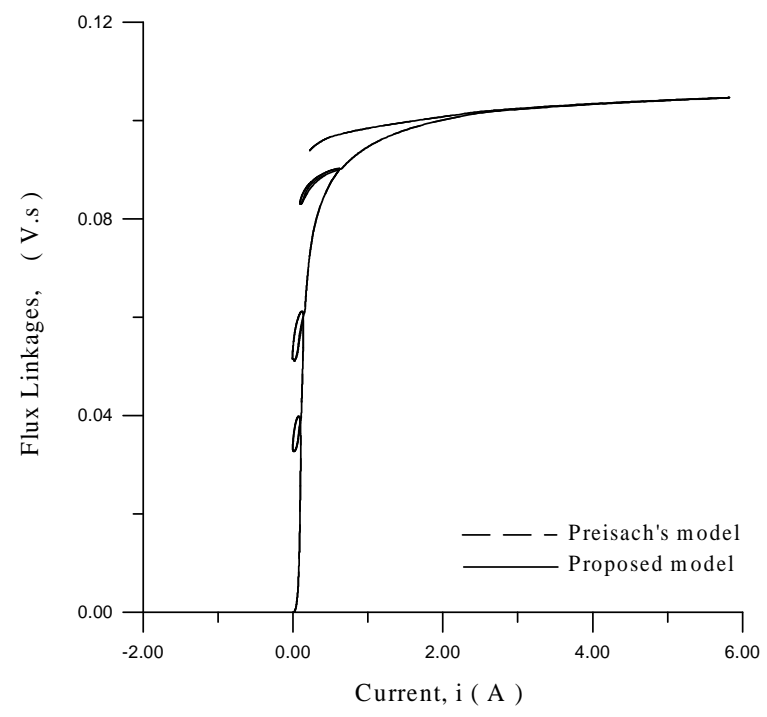

Figure 7: Hysteresis minor loops; $\boldsymbol{Z}=0.25+j 0.15 \Omega$.

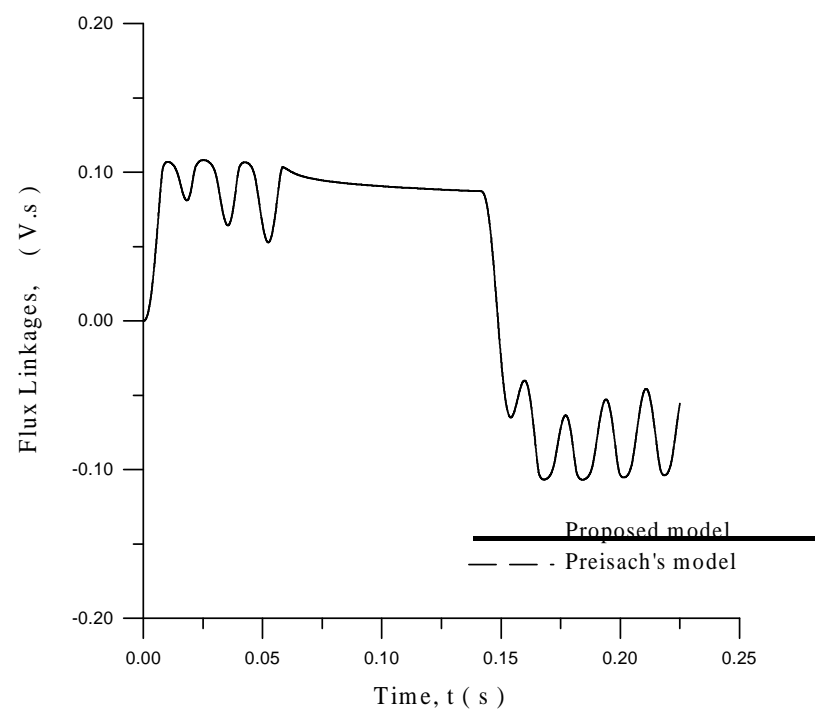

Figure 8: Flux linkages; $\theta_{1}=\theta_{2}=0^{\circ}, \boldsymbol{Z}=1.00+j 0.50 \Omega$.

obtained by another way, $i_{2}{ }^{*}$, is calculated as follows:

$$
\delta=100 \cdot \frac{i_{2}^{*}-i_{2}}{K_{f} I_{r}}
$$

Where, $I_{r}$ is the rated secondary current $(1 \mathrm{~A}) ; K_{f}$ is the overcurrent factor (20, according to international standards). The deviation $\delta$ is taken as a percentage of $K_{f} I_{r}$ because the $\mathrm{CT}$ performance must be evaluated in fault conditions.

The maximum deviations $\delta_{\max }$ occurred in the analyzed cases are summarized in table 1 .

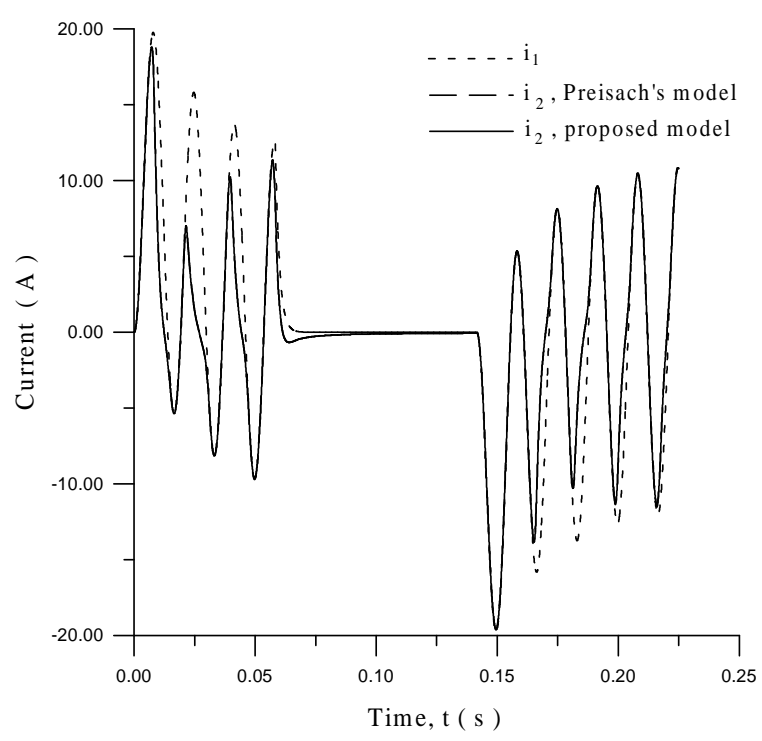

Figure 9: Currents; $\theta_{1}=\theta_{2}=0^{\circ}, \boldsymbol{Z}=1.00+j 0.50 \Omega$.

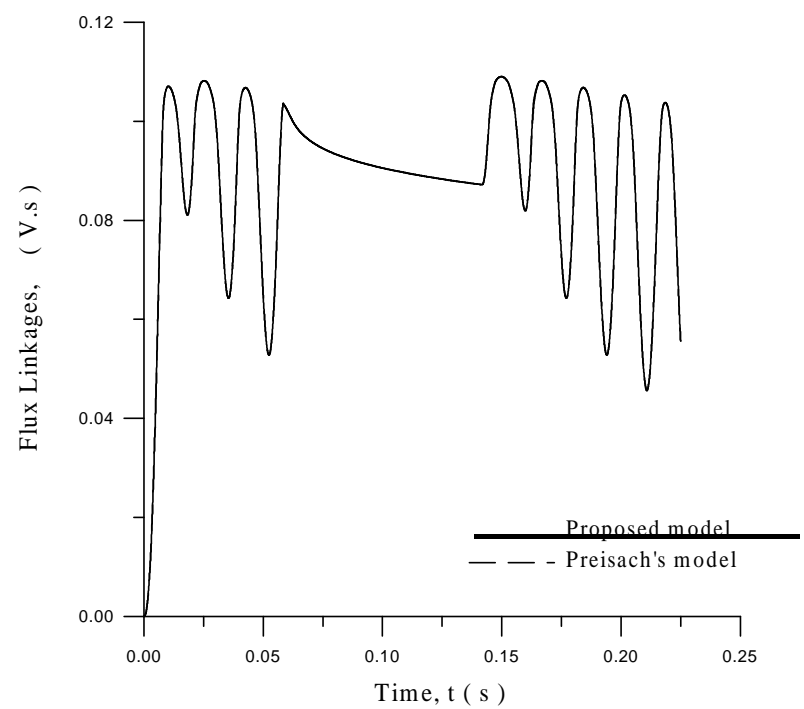

Figure 10: Flux linkages; $\theta_{1}=0^{\circ}, \theta_{2}=180^{\circ}, \boldsymbol{Z}=1.00+j$ $0.50 \Omega$.

Table 1: Maximum secondary current deviations

\begin{tabular}{|c|c|c|c|c|}
\hline Figure & Comparison & $\begin{array}{c}\text { CT burden } \\
(\Omega)\end{array}$ & $\begin{array}{c}\theta_{1}\left(\theta_{2}\right) \\
\left({ }^{\circ}\right)\end{array}$ & $\begin{array}{c}\delta_{\max } \\
(\%)\end{array}$ \\
\hline Figure 5 & $\begin{array}{c}\text { Proposed model vs } \\
\text { Experimental }\end{array}$ & $2.40+j 0.10$ & 0 & 3.1 \\
\hline Figure 6 & $\begin{array}{c}\text { Proposed model vs } \\
\text { Experimental }\end{array}$ & $1.52+j 1.70$ & 0 & 0.8 \\
\hline Figure 9 & $\begin{array}{c}\text { Proposed model vs } \\
\text { Preisach's model }\end{array}$ & $1.00+j 0.50$ & $0,(0)$ & 0.7 \\
\hline Figure 11 & $\begin{array}{c}\text { Proposed model vs } \\
\text { Preisach's model }\end{array}$ & $1.00+j 0.50$ & $0,(180)$ & 0.9 \\
\hline
\end{tabular}




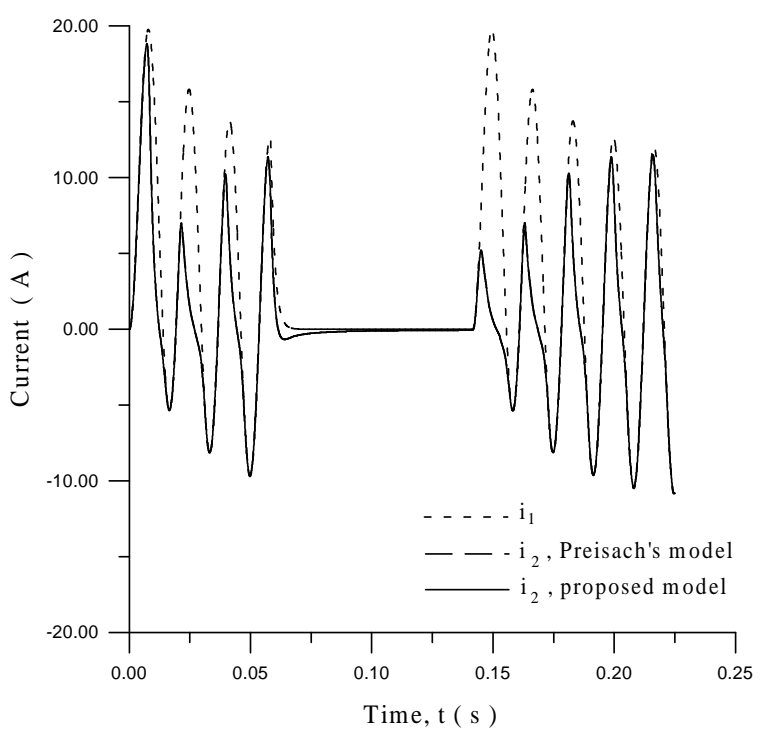

Figure 11: Currents; $\theta_{1}=0^{\circ}, \theta_{2}=180^{\circ}, \boldsymbol{Z}=1.00+j 0.50$ $\Omega$.

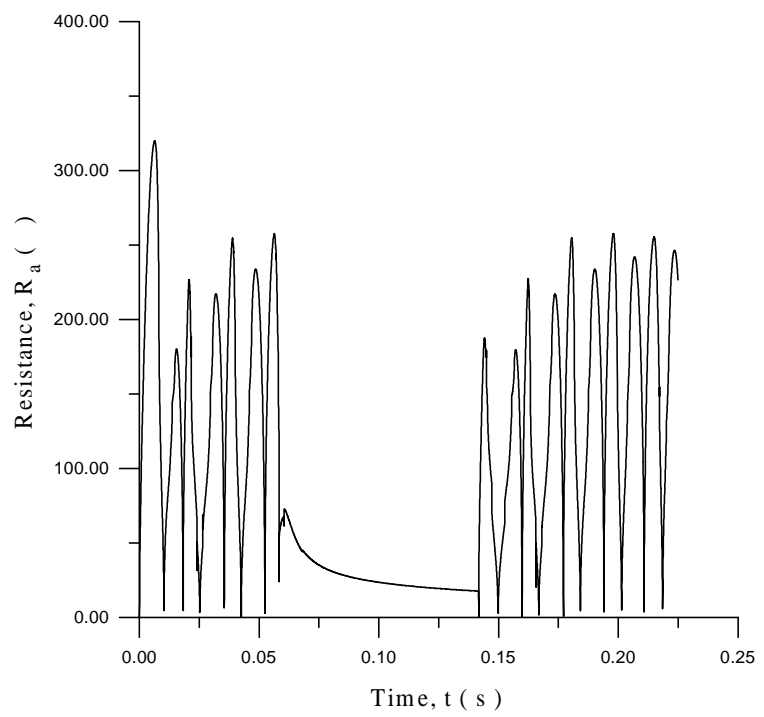

Figure 12: Anomalous losses resistance; $\theta_{1} 0^{\circ}, \theta_{2}=180^{\circ} . \boldsymbol{Z}$ $=1.00+j 0.50 \Omega$.

For the last case, the variation of the anomalous loss resistance with the time is shown in figure 12.

The results obtained through the proposed model were compared with those obtained using an easy model, in which the saturation curve is used to model the non-linear inductance. The total magnetic losses are represented by a linear resistance $(191.8 \Omega)$, determined from measurements of the losses with variable applied voltage. Although the $\lambda-i_{m}$ curve is singular, the iron core has a hysteretic behavior if the total

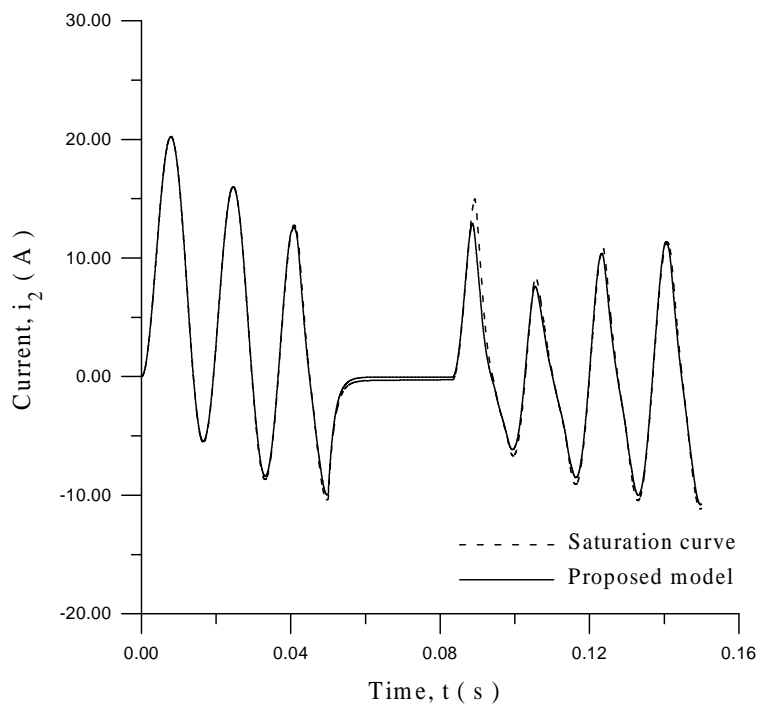

Figure 13: Currents; $\theta_{1}=0^{\circ}, \theta_{2}=0^{\circ}, \boldsymbol{Z}=0.40+j 0.15 \Omega$.

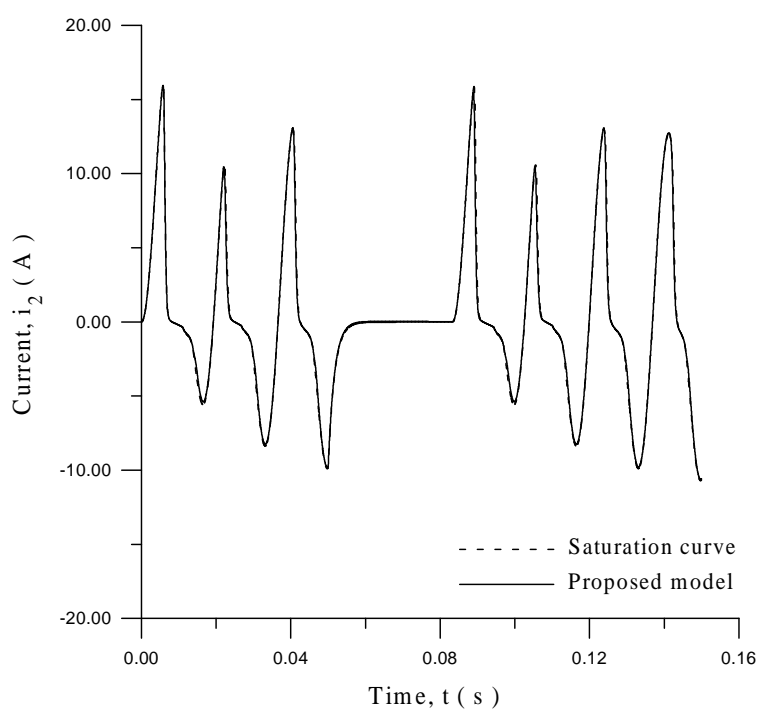

Figure 14: Currents; $\theta=0^{\circ}, \theta_{2}=0^{\circ}, \boldsymbol{Z}=2.25+j 0.15 \Omega$.

excitation current is taken into account.

It was considered three intervals of time: first closing $(3 \mathrm{cy}-$ cles, $\left.\theta_{1}=0^{\circ}\right)$, dead time ( 2 cycles) and reclosing $(4$ cycles, $\theta_{2}=0^{\circ}$ ). figure 13 and figure 14 illustrate the differences between the secondary currents, considering a burden of $0.40+$ $j 0.15 \Omega$ and $2.25+j 0.15 \Omega$, respectively.

It is observed that the differences are less pronounced for the higher impedance burden. When the impedance module increases, the magnetic core reaches a higher saturation level, and the hysteresis loop tends to the saturation curve. So, the magnetic excursions inside the major loop are not significant 
if compared with the large excursions in the saturated region.

\section{CONCLUSION}

A new current transformer model was presented, which includes the representation of the hysteresis and the dynamic losses in the core. The results obtained with the proposed hysteresis model are very close to the measurements, as well as to the Preisach's model. The model has a simple formulation, with easy understanding and implementation. The number of calculations is less than the Preisach model, because only simple algebraic equations are involved.

The graphical comparisons of the proposed hysteresis model with the model in which the saturation curve is used show that a more detailed iron core representation is necessary when moderate saturation levels are reached.

This method is valid for any toroidal non-gapped iron core $\mathrm{CT}$, regardless on size and rating. Moreover, it is suitable for multiple current transformers connected to the same burden. For applications in current transformers with complex magnetic cores, it is necessary additional developments.

The frequency limits of this model are the same ones established by standards like ANSI and IEC ( 25 up to $400 \mathrm{~Hz}$ ). For high frequency applications, it is necessary to include the distributed capacitances in an appropriate way.

Due the simplicity, the authors believe that this method may be used in other available tools for current transformers modeling, like EMTP and EMTDC.

The magnetic core model presented here is also useful for other purposes, such as studies of ferroresonance and inrush currents in power transformers.

\section{ACNOWLEDGMENT}

The authors would like to thank Prof. Damásio Fernandes Júnior of Universidade Federal de Campina Grande for his stimulating interest in this work and his suggestions.

\section{REFERENCES}

Bittanti, S., F. A. Cuzzola, F. Lorito \& G. Poncia (2001). Compensation of nonlinearities in the current transformer for the reconstruction of the primary current. IEEE Transactions on Control Systems Technology, Vol.9, $\mathrm{n}^{\circ} 4$, pp. 565-573.

Chaudhary, A. K. S., K. Tam \& A. G. Phadke (1994). Protection system representation in the Electromagnetic Transients Program. IEEE Transactions on Power Delivery,
Vol. $9, \mathrm{n}^{\circ} 2$, pp. $700-708$.

Conrad, T. \& D. Oeding (1987). A method to correct the distorted secondary currents of current transformers. Proceedings of PSCC, Vol. 2, $\mathrm{n}^{\circ}$ 3, pp. 311-315.

Elleuch, M. \& M. Poloujadoff, (1998). New transformer model including joint air gaps and lamination anisotropy. IEEE Transactions on Magnetics, Vol.34, $\mathrm{n}^{\circ} 5$, pp. 37013711.

Fiorillo, F. \& A. Novikov (1990) An improved approach to power losses in magnetic laminations under nonsinusoidal induction waveform. IEEE Transactions on Magnetics, Vol 26, n ${ }^{\circ}$ 5, pp. 2904-2910.

Frame, J. R.; N. Mohan \& T. Liu (1982). Hysteresis modeling in an electromagnetic transients program, IEEE Transactions on Power Apparatus and Systems, Vol. 101, $\mathrm{n}^{\circ} 9$, pp. 3403-3412.

Kang, Y. C, J. K. Park, S. H. Kang, A. T. Johns \& R. K. Aggarwal (1997). An algorithm for compensating secondary currents of current transformers, IEEE Transactions on Power Delivery, Vol. 12, $\mathrm{n}^{\circ}$ 1, pp. 116-124.

Kezunovic, M., C. W. Fromen \& F. Philips (1994). Experimental evaluation of EMTP-based current transformer models for protective relay transient study. IEEE Transactions on Power Delivery, Vol.9, $\mathrm{n}^{\circ}$ 1, pp. 405-412.

Locci, N. L. \& C. Muscas (2001). Hysteresis and eddy currents compensation in current transformers, IEEE Transactions on Power Delivery, Vol.16, $\mathrm{n}^{\circ} 2$, pp. 154-159.

Naidu, S. R. (1990). Simulation of the hysteresis phenomenon using Preisach's theory. IEE Proceedings, Vol. 137(A), $\mathrm{n}^{\circ} 2$, pp.73-79.

Poljak, M. \& M. Kolibas (1988). Computation of current transformer transient performance. IEEE Transactions on Power Delivery, Vol. 3, $\mathrm{n}^{\circ}$ 4, pp. 1816-1822.

Press, W. H., B. P. Flannery, S. A. Tewkolsky and W. T. Wetterling (1986). Numerical Recipes - The Art of Scientific Computing. Cambridge University Press.

Talukdar, S. R. \& J. R. Bailey (1976). Hysteresis model for system studies. IEEE Transactions on Power Apparatus and Systems, Vol.95, $\mathrm{n}^{\circ}$ 4, pp. 1429-1434.

Tellinen, J. (1998). The simple scalar model for magnetic hysteresis. IEEE Transactions on Magnetics, Vol.34, $\mathrm{n}^{o}$ 4, pp. 2200-2206.

Zhu, J. G., S. Y. R. Huy \& V. S. Ramsden (1993). Discrete modeling of magnetic cores including hysteresis, eddy current and anomalous losses, IEE Proceedings-A, vol. $140, \mathrm{n}^{\circ} 4$, pp. 317-322. 